\title{
Clustering of reported cases of leptospirosis among dogs in the United States and Canada
}

\author{
Michael P. Ward* \\ Department of Veterinary Pathobiology, School of Veterinary Medicine, Purdue University, \\ 1243 Veterinary Pathology Building, West Lafayette, IN 47907-1243, USA
}

Received 11 February 2002; accepted 2 September 2002

\begin{abstract}
A retrospective study was conducted to determine whether cases of leptospirosis diagnosed among dogs in the United States and Canada were clustered in time, space, or time-and-space. The study population was estimated-based on first examination of each dog-to consist of 1,035,366 dogs examined at 22 veterinary teaching hospitals between 1983 and 1998. The veterinary medicine database was searched for records of dogs in which a diagnosis of leptospirosis was made during the study period. The scan statistic (Poisson model) was used to examine for clustering of cases, using recorded date of diagnosis and the location of the veterinary teaching hospital at which the diagnosis was made.

Three-hundred and forty dogs diagnosed with leptospirosis were identified. Cases were clustered in the period of 1992-1998. A cluster of dogs diagnosed at three veterinary teaching hospitals located in the midwest of the United States was identified. The composition of this spatial cluster was influenced by the age and gender distribution of the population at-risk. A cluster of cases involving 143 dogs diagnosed with leptospirosis between 9 April 1993 and 14 December 1998 at six veterinary teaching hospitals located in the midwest of the United States was identified. This time-space cluster was not influenced by the age, gender or breed distribution of the population of dogs presented at veterinary teaching hospitals during the study period.

Leptospirosis was clustered during the 1990s in the midwest of the United States. Dog risk factors (age, gender, breed) do not explain the clustering that was observed. Leptospira serovar or environmental factors might be responsible for the clustering of canine leptospirosis in the United States and Canada.
\end{abstract}

(C) 2002 Elsevier Science B.V. All rights reserved.

Keywords: Leptospirosis; Canine; Cluster; Scan statistic; USA; Canada

\footnotetext{
*Tel.: +1-765-494-5796; fax: +1-765-494-9830.

E-mail address: mpw@vet.purdue.edu (M.P. Ward).
} 


\section{Introduction}

Leptospirosis occurs in many animal species and humans. In dogs, it typically presents as acute renal and/or hepatic failure with or without coagulation abnormalities (Harkin and Gartrell, 1996; Birnbaum et al., 1998). Many different Leptospira serovars have been described. The epidemiology of leptospirosis is characterized by a primary host species that acts as the reservoir for each serovar, maintaining the disease. Localized outbreaks of leptospirosis can occur when secondary host species are infected through environmental contamination (Bolin, 1996).

Leptospirosis due to Leptospira interrogans serovars canicola and icterohaemorrhagiae has been reported in dogs in the United States and Canada for more than 100 years (Bolin, 1996). Dogs are the reservoir species for L. interrogans serovar canicola, and rats are the reservoir species for $L$. interrogans serovar icterohaemorrhagiae. More recently, leptospirosis caused by other serovars such as L. interrogans serovar bratislava, Leptospira kirschneri serovar grippotyphosa and L. interrogans serovar Pomona have been described. Other serovars pathogenic for dogs included australis, autumnalis, ballum, bataviae and hardjo. These serovars have a range of reservoir species, including voles, raccoons, skunks, opossums, mice, pigs and cattle (Hanson, 1982; Bolin, 1996; Wohl, 1996; Birnbaum et al., 1998; Adin and Cowgill, 2000; Sykes, 2001).

Ward et al. (2002) examined the prevalence of leptospirosis in dogs presented to veterinary teaching hospitals in the United States and Canada between 1970 and 1998. Between 1970 and 1982, the prevalence of leptospirosis decreased. However, between 1983 and 1998 the prevalence increased significantly $(P<0.01)$, an annual increase of 1.2 cases per 100,000 dogs examined. Age, gender, and breed were risk factors for leptospirosis. Dogs in herding, working, and gundog breed categories and sexually intact male dogs appeared to be at the greatest risk of being diagnosed with leptospirosis.

Reasons for increased prevalence of leptospirosis during the 1980s and 1990s are unclear. Leptospirosis caused by serovars canicola and icterohaemmorrhagiae has been controlled by a bivalent vaccine, introduced in the early 1970s (Hanson, 1982; Bolin, 1996; Sykes, 2001). Dogs that received the bivalent vaccine were not protected from infection with serovars such as bratislava, pomona and grippotyphosa (Hanson, 1982; Rentko et al., 1992; Bolin, 1996; Birnbaum et al., 1998), because vaccine protection is serovar-specific. In addition, urbanization of rural areas (increasing during the 1980s and 1990s) might provide more opportunity for contact between dogs and wildlife (such as skunks, raccoons, and opossums, which serve as reservoirs for some of these serovars), and for contact between dogs and livestock or livestock waste (Hanson, 1982; Bolin, 1996; Birnbaum et al., 1998). Identifying and describing clustering of leptospirosis during a period of increasing prevalence (1983-1998) can provide information with which to develop hypotheses to explain the apparent changing epidemiology of canine leptospirosis in the United States and Canada. The objectives of this study were to determine if cases of leptospirosis diagnosed at veterinary teaching hospitals in the United States and Canada were clustered in time, space, and time-and-space, to describe the clusters detected, and determine if identified clusters could be explained by the age, gender or breed distribution of the population at-risk. 


\section{Materials and methods}

\subsection{Data source}

The selection of records used in this study has been described previously (Ward et al., 2002). Briefly, the veterinary medical database (VMDB) was used to identify records of dogs examined at veterinary teaching hospitals in the United States and Canada between 1 January 1983 and 31 December 1998. Records of dogs examined each year at 22 veterinary teaching hospitals located at schools and colleges of veterinary medicine (Table 1) in which a diagnosis of leptospirosis had been made (VMDB diagnosis code 010017200) were identified. Information was extracted from each record on veterinary teaching hospital, date of diagnosis, age at diagnosis ( $<2$ weeks, 2-8 weeks, 9-24 weeks, 25 weeks to 1 year, 1.1-1.9 years, 2-3.9 years, 4-6.9 years, 7-10 years, 10.1-15 years, >15 years, unknown), gender (male, male castrated, male unknown, female, female spayed, female unknown, unknown) and breed (purebred (name of breed was recorded), mixed breed, unknown). For each veterinary teaching hospital included in the study, information on the population

Table 1

Number of cases of leptospirosis diagnosed among dogs examined between 1983 and 1998 at 22 veterinary teaching hospitals in the United States and Canada that participated in the VMDB

\begin{tabular}{|c|c|c|c|c|c|c|c|}
\hline \multicolumn{2}{|c|}{ Teaching hospital } & \multirow{2}{*}{$\begin{array}{l}\text { Reporting } \\
\text { period }\end{array}$} & \multirow{2}{*}{$\begin{array}{l}\text { Total } \\
\text { years }\end{array}$} & \multirow{2}{*}{$\begin{array}{l}\text { Total no. } \\
\text { of dogs }\end{array}$} & \multicolumn{3}{|c|}{ Leptospirosis } \\
\hline Number & Name & & & & No. & $\begin{array}{l}\text { No. per } \\
100,000 \text { dogs }\end{array}$ & $95 \% \mathrm{CI}$ \\
\hline 1 & Michigan State University & 1983-1998 & 16 & 103,193 & 73 & 71 & 56,88 \\
\hline 2 & University of Missouri & 1983-1998 & 16 & 46,705 & 5 & 11 & 4,24 \\
\hline 3 & University of Minnesota & 1983-1998 & 16 & 59,938 & 6 & 10 & 4,21 \\
\hline 4 & Iowa State University & 1983-1998 & 16 & 49,921 & 49 & 98 & 73,129 \\
\hline 5 & Cornell University & 1983-1991 & 9 & 39,780 & 37 & 93 & 66,127 \\
\hline 6 & University of Guelph & 1983-1991 & 9 & 23,526 & 1 & 4 & 0,21 \\
\hline 7 & Purdue University & 1983-1998 & 16 & 44,389 & 24 & 54 & 35,79 \\
\hline 8 & University of Georgia & 1983-1998 & 16 & 65,557 & 24 & 37 & 24,54 \\
\hline 9 & University of California & $1983-1987$ & 5 & 19,967 & 2 & 10 & 2,33 \\
\hline 10 & Ohio State University & 1983-1989 & 7 & 45,505 & 5 & 11 & 4,24 \\
\hline 11 & Kansas State University & 1983-1993 & 11 & 33,964 & 0 & - & - \\
\hline 12 & University of Illinois & 1983-1998 & 16 & 62,965 & 33 & 52 & 37,73 \\
\hline 13 & University of Saskatchewan & 1983-1989 & 7 & 15,626 & 0 & - & - \\
\hline 14 & Colorado State University & 1983-1998 & 16 & 92,929 & 2 & 2 & 0,7 \\
\hline 15 & Auburn University & 1983-1998 & 16 & 56,450 & 8 & 14 & 7,27 \\
\hline 16 & Texas A\&M University & 1983-1998 & 16 & 64,669 & 29 & 45 & 31,64 \\
\hline 17 & University of Tennessee & 1983-1996 & 14 & 43,603 & 6 & 14 & 6,29 \\
\hline 18 & Louisiana State University & 1983-1994 & 12 & 31,625 & 4 & 13 & 4,30 \\
\hline 19 & $\begin{array}{l}\text { Virginia-Maryland Regional } \\
\text { College of Veterinary Medicine }\end{array}$ & 1988-1994 & 7 & 11,675 & 2 & 17 & 3,56 \\
\hline 20 & University of Wisconsin & 1987-1997 & 11 & 39,463 & 23 & 58 & 38,86 \\
\hline 21 & University of Pennsylvania & 1986-1994 & 9 & 49,226 & 6 & 12 & 5,25 \\
\hline \multirow[t]{2}{*}{22} & University of Florida & 1984-1994 & 11 & 34,690 & 1 & 3 & 0,14 \\
\hline & Total & & 272 & $1,035,366$ & 340 & 33 & 29,36 \\
\hline
\end{tabular}


at-risk (the total number of dogs examined during each year, and the number of dogs in each age, gender and breed category) was also extracted from the VMDB. The annual population at-risk for each hospital was estimated by the number of dogs examined each year at that hospital, with only an individual dog's first examination during each year used in the estimate-repeat visits being excluded from the estimate of population at-risk. The total population at-risk during the study period for each hospital was estimated by the number of dogs examined at the hospital, with only an individual dog's first examination during its life used in the estimate-repeat visits also being excluded from the estimate. The annual and the total populations at-risk for all hospitals were estimated as the sums of hospital-specific annual populations at-risk and total populations at-risk, respectively. In all cases, the population at-risk was number of dogs.

\subsection{Data analysis}

The prevalence of leptospirosis was calculated for the study period, and for each hospital included in the study. Confidence intervals were estimated for prevalences, assuming leptospirosis occurrence is described by the Poisson distribution (Kahn and Sempos, 1989). Clustering of leptospirosis cases in time, space, and time-and-space was analyzed using the scan statistic (Kulldorff et al., 1998a). A one-dimensional scan statistic has been used to study disease clusters in time and a two-dimensional scan statistic has been proposed for spatial disease clusters (Naus, 1965; Kulldorff, 1997). Kulldorff et al. (1998a) proposed extending the use of the scan statistic (defined by a cylindrical window with a circular geographic base and height corresponding to time) for time-space clustering. Clustering of disease can occur independently in temporal and spatial dimensions. In addition, clustering can occur in time-and-space, without clustering being apparent in either the temporal or spatial dimensions (Ward and Carpenter, 2000a,b). Because the study was undertaken to generate hypotheses regarding causes of canine leptopsirosis, the data therefore were examined for clustering in time, space, and time-and-space. The scan statistic is a clusterdetection test-able to both identify and test the statistical significance of specific disease clusters. This statistic has several potential advantages, including the ability to include covariates in the analysis through the method of indirect standardization, application for analyzing clustering in heterogeneous populations, and adjustment for multiple testing (Kulldorff et al., 1998a,b). The scan statistic allows the number of cases of disease expected within the scanning window (as it is moved across each centroid) to be distributed as either Poisson (rate data) or Bernoulli (case-control data). Using a Poisson model, the number of cases of disease within each study area (e.g. veterinary teaching hospital source population) is assumed to be proportional to the area's population size (animals or animal-years atrisk). The distribution of occurrence of leptospirosis was assumed to be Poisson and the observed number of cases defined the distribution. The population at-risk used in analyses was the annual number of dogs examined at each veterinary teaching hospital. The data set was scanned for clusters with only high rates of leptospirosis (equivalent to a one-sided statistical test) in time, space and/or time-and-space. The data set was scanned for clusters in spatial and temporal dimensions ranging from 0 to $50 \%$ of the total population at-risk (up to $50 \%$ of the study area - approximately 2.9 million $\mathrm{km}^{2}$ - and $50 \%$ of the study period96 months) because a cluster of size larger than $50 \%$ would indicate hospitals with 
exceptionally low occurrences of leptospirosis outside the scanning window rather than hospitals with exceptionally high leptospirosis occurrences within the window (Kulldorff et al., 1998b). In time and time-space analyses, the increasing trend in leptospirosis prevalence during the study period (5.8\% per year; Ward et al., 2002) was adjusted so that a cluster occurring at the end of the study period would not be detected simply because of the long-term trend in the data set. Clusters were identified by the scanning window associated with the maximum-likelihood, and a likelihood-ratio test statistic was calculated. The distribution of the likelihood-ratio and its corresponding $P$ was obtained by Monte-Carlo simulation-randomly generating 999 replications of the data set under the null hypothesis. For hypothesis testing, the test statistic was calculated for each random replication as well as for the study data set (a total of 1000 statistics) - and if the latter was the most extreme $5 \%$ of all test statistics calculated (that is, ranked 50th or higher), then the hypothesis test was significant at $P=0.050$. Thus, the problem of performing multiple tests using many scanning windows was addressed and type-I error was restricted to $5 \%$. The number of replications chosen was considered to provide moderate statistical power whilst minimizing computing time (Kulldorff et al., 1998b).

All crude analyzes were re-analyzed adjusting separately for the age, gender and breed distribution of all dogs examined at each veterinary teaching hospital during each year of the study. Adjustments were made using indirect standardization, in which the expected number of cases of leptospirosis were calculated based on the frequency distribution of age, gender or breed for dogs examined at each hospital, in each year and for each combination of hospital and year. Age was re-categorized as <1, 1-1.9, 2-3.9, 4-6.9, 7-10 and $>10$ years to produce a more uniform distribution (because age was right-skewed) and, therefore, more efficient indirect standardization. Records in which age, gender or breed were unknown were excluded from each covariate-specific analysis. All calculations were performed using SaTScan software version 2.1 (Kulldorff et al., 1998b). Rainfall data were obtained for the cities in which veterinary teaching hospitals which were included in identified clusters are located, for the period in which clustering of cases of leptospirosis were diagnosed (National Climate Data Centre, US Monthly Precipitation for Cooperative \& NWS Sites, http://lwf.ncdc.noaa.gov/oa/climate/online/coop-precip.html; National Snow and Ice Data Center, 2000; Adjusted Monthly Precipitation, Snowfall and Rainfall for Canada (1874-1990). EOSDIS NSIDC Distributed Active Archive Center, University of Colorado at Boulder, http://nsidc.org/data/docs/daac/nsidc0072_canadian _precip.gd.html). Mean monthly rainfall was compared to the long-term mean monthly rainfall recorded at these same locations. The effect of the adjustments made were assessed by comparing relative risk estimates (crude relative risk - adjusted relative risk)/crude relative risk. A reduction in estimated relative risk $>10 \%$ was considered substantial and to suggest an important cause of the clustering detected.

\section{Results}

The 22 participating veterinary teaching hospitals in the United States and Canada submitted to the VMDB records on 1,067,214 dogs examined during the study period (Table 1). Ten teaching hospitals submitted records to the VMDB during each year of the 
study period. Mean number of years of reporting was 12. A total of 340 cases of leptospirosis (33 per 100,000 dogs examined; 95\% CI, 29-36 cases per 100,000 dogs) was identified from the VMDB between 1983 and 1998. The greatest number of cases (73) was diagnosed at Michigan State University (71 per 100,000 dogs examined; 95\% CI, 5688 cases per 100,000). No cases were diagnosed at Kansas State University and the University of Saskatchewan. Age, gender or breed was not recorded for 15,101 (1.41\%), $13,922(1.30 \%)$ and $113(0.01 \%)$ of the dogs included in the study, respectively. These records were excluded from age, gender and breed-specific analyzes. The overall age structure $(<1,1-1.9,2-3.9,4-6.9,7-10,>10$ years $)$ of the population was $21,11,17,19,16$ and $17 \%$, respectively. The overall gender structure (male, male castrated, female, female spayed) of the population was 31, 16, 22 and 30\%, respectively. The greatest proportions of dogs were in mixed (24\%), Labrador Retriever (7\%), Golden Retriever (5\%), German Shepherd (5\%) and American Cocker Spaniel (4\%) breed categories.

Significant $(P=0.001)$ clustering of leptospirosis in time and in space was detected. The temporal cluster consisted of 235 cases ( 54 per 100,000 dogs; $95 \%$ CI, 48-61 cases per 100,000) diagnosed between 3 July 1992 and 14 December 1998 (Table 2). A spatial cluster consisting of 120 cases (64 per 100,000 dogs; 95\% CI, 53-76 cases per 100,000) of leptospirosis was identified and included veterinary teaching hospitals located at Michigan State and Purdue Universities and the University of Wisconsin (Table 3). Significant $(P=0.001)$ clustering of leptospirosis in time-and-space was detected. The cluster of leptospirosis consisted of cases diagnosed between 9 April 1993 and 14 December 1998 at veterinary teaching hospitals located at the Universities of Wisconsin, Minnesota, Missouri, and Iowa State, Michigan State and Purdue Universities. This cluster consisted of 143 cases (91 per 100,000 dogs; 95\% CI, 77-107 cases per 100,000). Based on the Poisson distribution (population of dogs at-risk), only 52 cases of leptospirosis were expected (Table 4).

Adjusting for the age, gender and breed distribution of the population at-risk made no substantial difference (change in relative risk $<10 \%$ ) to the temporal cluster of leptospirosis

Table 2

Temporal clustering of cases of canine leptospirosis diagnosed at veterinary teaching hospitals in the United States and Canada between 1 January 1983 and 31 December 1998, and adjusting for age (<2, 2-3.9, 4-6.9, 7$10,>10$ years), gender (male, male castrated, female, female spayed) and breed distribution of the population of dogs at-risk

\begin{tabular}{|c|c|c|c|c|c|c|c|}
\hline \multirow[t]{2}{*}{ Covariate } & \multicolumn{2}{|l|}{ Time period } & \multicolumn{2}{|c|}{$\begin{array}{l}\text { Number of cases of } \\
\text { leptospirosis diagnosed }\end{array}$} & \multirow[t]{2}{*}{ Rate $^{\mathrm{a}}$} & \multirow[t]{2}{*}{$\mathrm{RR}^{\mathrm{b}}$} & \multirow[t]{2}{*}{$\operatorname{LLR}^{\mathrm{c}}$} \\
\hline & Start date & End date & Expected & Observed & & & \\
\hline- & 3 July 1992 & 14 December 1998 & 235 & 142 & 54 & 1.65 & 51.4 \\
\hline Age & 1 January 1993 & 31 December 1998 & 214 & 130 & 43 & 1.65 & 42.9 \\
\hline Gender & 1 January 1993 & 31 December 1998 & 214 & 133 & 40 & 1.61 & 39.9 \\
\hline Breed & 1 January 1993 & 31 December 1998 & 216 & 134 & 53 & 1.61 & 40.2 \\
\hline
\end{tabular}

${ }^{\text {a }}$ Cases observed per 100,000 dogs at-risk.

${ }^{\mathrm{b}}$ Ratio of the number of cases observed in the identified cluster and the number of cases expected in the population, assuming that cases of diagnosed leptospirosis are Poisson distributed.

${ }^{\mathrm{c}}$ Log likelihood-ratio statistic. 
Table 3

Spatial clustering of cases of canine leptospirosis diagnosed at veterinary teaching hospitals (VTHs) in the United States and Canada between 1 January 1983 and 31 December 1998, and adjusting for age $(<2,2-3.9,4$ $6.9,7-10,>10$ years), gender (male, male castrated, female, female spayed) and breed distribution of the population of dogs at-risk

\begin{tabular}{|c|c|c|c|c|c|c|}
\hline \multirow[t]{2}{*}{ Covariate } & \multirow[t]{2}{*}{$\mathrm{VTH}^{\mathrm{a}}$} & \multicolumn{2}{|c|}{$\begin{array}{l}\text { Number of cases of } \\
\text { leptospirosis diagnosed }\end{array}$} & \multirow[t]{2}{*}{ Rate $^{b}$} & \multirow[t]{2}{*}{$\mathrm{RR}^{\mathrm{c}}$} & \multirow[t]{2}{*}{$\operatorname{LLR}^{\mathrm{d}}$} \\
\hline & & Expected & Observed & & & \\
\hline- & $1,7,20$ & 61 & 120 & 64 & 1.95 & 28.4 \\
\hline Age & $1,5,6$ & 53 & 108 & 64 & 2.03 & 27.4 \\
\hline Gender & $1,5,6$ & 54 & 110 & 65 & 2.04 & 28.3 \\
\hline Breed & $1,7,20$ & 62 & 120 & 63 & 1.92 & 27.3 \\
\hline
\end{tabular}

\footnotetext{
${ }^{\text {a }}$ See Table 1.

${ }^{\mathrm{b}}$ Cases observed per 100,000 dogs at-risk.

${ }^{c}$ Ratio of the number of cases observed in the identified cluster and the number of cases expected in the population, assuming that cases of diagnosed leptospirosis are Poisson distributed.

${ }^{\mathrm{d}}$ Log likelihood-ratio statistic.
}

diagnoses detected in the study period (Table 2). However, adjusting for the age and the gender distribution of the population at-risk did result in a change to the spatial cluster of cases of leptospirosis that was identified: a new cluster was identified that included cases diagnosed at veterinary teaching hospitals located at Michigan State and Cornell Universities and the University of Guelph (Table 3). Adjusting for the breed distribution of the population at-risk made no substantial difference to the spatial cluster of leptospirosis diagnoses detected (Table 3). Also, adjusting for the age, the gender and the breed distribution of the population at-risk made no substantial difference to the cluster of

Table 4

Temporal and spatial clustering of cases of canine leptospirosis diagnosed at veterinary teaching hospitals (VTHs) in the United States and Canada between 1 January 1983 and 31 December 1998, and adjusting for age $(<2,2-3.9,4-6.9,7-10,>10$ years), gender (male, male castrated, female, female spayed) and breed distribution of the population of dogs at-risk

\begin{tabular}{|c|c|c|c|c|c|c|c|c|}
\hline \multirow[t]{2}{*}{ Covariate } & \multirow[t]{2}{*}{ Start } & \multirow[t]{2}{*}{ End } & \multirow[t]{2}{*}{$\mathrm{VTH}^{\mathrm{a}}$} & \multicolumn{2}{|c|}{$\begin{array}{l}\text { Number of cases } \\
\text { of leptospirosis } \\
\text { diagnosed }\end{array}$} & \multirow[t]{2}{*}{ Rate $^{b}$} & \multirow[t]{2}{*}{$\mathrm{RR}^{\mathrm{c}}$} & \multirow[t]{2}{*}{$\operatorname{LLR}^{\mathrm{d}}$} \\
\hline & & & & Expected & Observed & & & \\
\hline- & 9 April 1993 & 14 December 1998 & $1-4,7,20$ & 52 & 143 & 91 & 2.77 & 70.8 \\
\hline Age & 9 April 1993 & 14 December 1998 & $1-4,7,20$ & 52 & 143 & 88 & 2.77 & 71.2 \\
\hline Gender & 9 April 1993 & 14 December 1998 & $1-4,7,20$ & 52 & 142 & 88 & 2.75 & 69.4 \\
\hline Breed & 9 April 1993 & 14 December 1998 & $1-4,7,20$ & 54 & 143 & 87 & 2.66 & 66.2 \\
\hline
\end{tabular}

\footnotetext{
${ }^{\text {a }}$ See Table 1.

${ }^{\mathrm{b}}$ Cases observed per 100,000 dogs at-risk.

${ }^{c}$ Ratio of the number of cases observed in the identified cluster and the number of cases expected in the population, assuming that cases of diagnosed leptospirosis are Poisson distributed.

${ }^{\mathrm{d}}$ Log likelihood-ratio statistic.
} 
leptospirosis diagnoses in time-and-space that was identified (Table 4). No secondary clusters associated with increased risk of leptospirosis were identified in any of the analyzes. The mean (S.D.) monthly rainfall recorded during the period April 1993December 1998 at the six cities where veterinary teaching hospitals included in the timespace cluster are located was $79 \mathrm{~mm}$ (56), compared to a long-term (1900-1998) mean monthly rainfall at these locations of $70 \mathrm{~mm}(50)$.

\section{Discussion}

Results of this study suggest that, during the period of increasing prevalence of leptospirosis in the United States and Canada, cases were diagnosed at a higher rate than expected between 1993 and 1998 at veterinary teaching hospitals located in the midwest of the United States. Changes in the age-, gender- and breed-distribution of dogs presented to veterinary teaching hospitals in the United States and Canada do not explain the clustering detected. Possible explanations for the clustering detected include a shift in the serovars causing disease and changes in the exposure of dogs to Leptospira serovars, climatic factors and referral, diagnostic and reporting biases.

The serovars causing leptospirosis were not reported in records submitted to the VMDB. Leptospirosis caused by one or more serovars could be clustered, and responsible for the overall clustering detected in this study, while cases of leptospirosis caused by other serovars might have occurred either sporadically or uniformly during the study period and study area. A bivalent vaccine for protection of dogs against leptospirosis due to serovars canicola and icterohaemorrhagiae has been widely available since at least the early 1970s. It has been suggested that, because of the introduction of this bivalent vaccine, leptospirosis caused by serovars canicola and icterohaemorrhagiae has decreased in the United States and Canada (Wohl, 1996; Sykes, 2001). However, cases caused by infection with serovars bratislava, grippotyphosa and pomona might have increased. Serovars canicola and icterohaemorrhagiae are maintained by dogs and rats, respectively. Direct transmission of Leptospira serovars between infected dogs is considered to be infrequent (Wohl, 1996), so in a stable system, increasing leptospirosis prevalence cannot be explained adequately by increasing $L$. interrogans serovar canicola infection. Rats are a ubiquitous reservoir of $L$. interrogans serovar icterohaemorrhagiae, and dogs are unlikely to have been more often exposed during the 1990s and in the midwest region of the United States. The clustering of leptospirosis detected in this study might be attributed to a decrease in the proportion of the dog population in the midwest region of the United States vaccinated against leptospirosis, using the bivalent vaccine. The Leptospira bacterin is thought to cause a high rate of adverse reactions and to be immunosuppressive in dogs < 16 weeks of age (Markey, 1995; Paul and Wolf, 1999), and some veterinarians have suggested there is little indication for its use (Paul and Wolf, 1999). However, recent case studies conducted on hospital populations located in different regions of the United States and Canada have failed to detect substantial proportions of dogs infected with serovars canicola or icterohaemorrhagiae (Brown et al., 1996; Harkin and Gartrell, 1996; Birnbaum et al., 1998; Adin and Cowgill, 2000). Therefore, infection by these serovars is unlikely to explain the increasing prevalence of leptospirosis in the 1990s (Bolin, 1996). Case studies of leptospirosis conducted in the 
1990s on populations of dogs presented to hospitals located in New York, California, Georgia, New Jersey, Michigan, Ontario and Québec have found L. interrogans serovar bratislava, L. kirschneri serovar grippotyphosa and/or L. interrogans serovar pomona the dominant serovars causing leptospirosis (Harkin and Gartrell, 1996; Birnbaum et al., 1998; Prescott et al., 1999; Adin and Cowgill, 2000; Ribotta et al., 2000). In a survey of 243 pet dogs presented to a veterinary hospital located in central Indiana during 2001 (LT Glickman, unpublished data), prevalence of antibodies (microagglutination test titer $\geq 1: 200$ ) was the highest for serovars grippotyphosa $(5.3 \%)$ and pomona $(2.1 \%)$. Prevalence of antibodies to serovars canicola and icterohaemorrhagiae were 0.4 and $0.8 \%$, respectively. Known reservoirs for serovars bratislava, pomona and grippotyphosa include pigs and possibly horses, pigs, cattle, skunks and opossums, and raccoons, skunks and opossums, respectively. If the clustering detected is due to increasing infection by these serovars, it suggests that the dog population of the midwest region of the United States has been exposed to these reservoir species or to environments contaminated by these species more frequently than other populations during the 1990s. Harkin and Gartrell (1996) and Bolin (1996) have suggested that housing developments that encroach on areas previously habited only by wildlife lead to greater exposure of dogs to Leptospira serovars that have wildlife species as their maintenance hosts. Supporting this hypothesis is the observation made by Rentko et al. (1992) that the prevalence of leptospirosis due to serovars pomona and grippotyphosa diagnosed at Tufts University Veterinary Teaching Hospital increased when the hospital was relocated from an urban environment to a rural location in central Massachusetts. Raccoons have been suggested as the source of infection for cases of leptospirosis due to serovar grippotyphosa in Georgia (Brown et al., 1996). Contamination of land with waste from swine production is another possible explanation for clustering, if serovars bratislava and/or pomona are responsible for the increasing prevalence of leptospirosis in the United States and Canada.

Flooding has been associated with outbreaks of leptospirosis in humans and animals (Rentko et al., 1992); exposure to water contaminated with the urine of infected animals is an occupational hazard for sanitary and agricultural workers, and also a recreational hazard for humans (Hanson, 1982; American Public Health Association, 2000). Rainfall can predict the number of dogs with leptospirosis (Adin and Cowgill, 2000). Environmental changes that result in a greater proportion of land being regularly flooded (for example, a period of above-average rainfall) might be an explanation for clustering of leptospirosis. In the present study, locations included in the time-space cluster had a higher mean monthly rainfall during the period when cases of leptospirosis were diagnosed, compared to the long-term mean monthly rainfall at these locations. This suggests that the occurrence of leptospirosis is associated with rainfall. If this is a causal association, preventing exposure of dogs to areas of land that have been flooded recently and to natural water features that are contaminated with Leptospira serovars could reduce the occurrence of leptospirosis.

The VMDB - a secondary data source-was used. Data analysis was limited to covariates recorded in the VMDB, and study findings might be subjected to bias in recorded information on risk factors and diagnosis of leptospirosis, as well as referral bias. It is not possible to assess the effect of potential misclassification of covariates on the accuracy of study results. However, the use of broad classes to categorize age should have minimized misclassification bias, and misclassification of gender or breed is unlikely in a 
veterinary teaching hospital. All dog records in the VMDB in which a diagnosis of leptospirosis was made between 1983 and 1998 were included. Because of the steps involved in making a diagnosis of leptospirosis (clinical examination and biochemical, hematologic, and serologic testing), it is unlikely that a dog would be diagnosed falsely with leptospirosis. It is more likely that leptospirosis was not diagnosed in some dogs with a mild form of the disease. As proposed by Ward et al. (2002), this source of bias is unlikely to be substantial because the number of dogs with undiagnosed leptospirosis is probably a very small proportion of all dogs examined at veterinary teaching hospitals. Procedures used to diagnose leptospirosis in dogs examined at veterinary teaching hospitals appear to have remained constant between reports of leptospirosis published in the 1980s (Baldwin and Atkins, 1987) and more recently published reports (Adin and Cowgill, 2000). However, awareness of leptospirosis among clinicians might have changed (Baldwin and Atkins, 1987; Brown et al., 1996; Birnbaum et al., 1998; Ribotta et al., 2000). Awareness of canine leptospirosis probably increased among clinicians during the 1990s, but it seems unlikely that awareness would be greater in the midwest region of the United States and could explain the clustering detected in this study. All studies using hospitalrecorded data are subject to referral bias. Although veterinary teaching hospitals in the United States and Canada general have a community-practice component, they are also referral hospitals and dogs examined at these hospitals might not be representative of all dogs in the target population: pet dogs living in the United States and Canada. In particular, dogs with leptospirosis might be more likely to be examined at a veterinary teaching hospital than dogs receiving routine veterinary care. However, if it can be assumed that referral bias was equivalent between hospitals included in this study — and constant during the study period-referral bias is irrelevant because disease-clustering analyses are based on comparisons within time, space or time-and-space dimensions. Similarly, the data analyzed do not need to be representative of the target population, assuming that referral and diagnostic biases are constant between study sites and during the study period. From data available in the VMDB, it is not possible to estimate hospital- or year-specific referral bias, or diagnostic bias.

Statistical tests are necessary to investigate temporal and spatial clustering of disease objectively (Ward and Carpenter, 2000a,b). The scan statistic can accommodate uneven population densities, is flexible with respect to data format (rate or case-control data, individual or aggregated data) and size of the scanning window used, and multiple testing can be avoided through a Monte-Carlo approach. Few examples of investigating temporal, spatial and time-space clustering of disease exist in the veterinary literature (Ward and Carpenter, 2000a,b), and apparently only one report has been published using information from the VMDB (O'Brien et al., 2000). Information on clustering of leptospirosis in dogs provides clues to possible reasons for the apparent epidemic of leptospirosis in the United States and Canada. Clustering occurred in the latter part of the study period (1993-1998), and clustering involved hospitals located in the midwest of the United States. When clustering in time-and-space was examined, clusters of leptospirosis were identified during the same time period and in the same geographical region, but included more hospital locations. This illustrates the need to identify clustering in time, space, and time-and-space: results suggest that clustering of leptospirosis within the 1990s was more widespread in the midwest region—including hospitals located in Missouri, Iowa and Minnesota—-than if the 
data had only been scanned for clusters in time and clusters in space. The clustering identified in time-and-space (RR 2.77) provides more compelling evidence (than clustering in only time (RR 1.65) or in only space (RR 1.95)) - that risk factors for leptospirosis might also operate at a regional-level. If these risk factors can be modified or avoided, the impact of leptospirosis on the canine population of the United States and Canada can be reduced. Characterization of serovar-specific clustering of leptospirosis cases would enable the reasons for clustering to be better understood-assisting in the development of more targeted control programs. Planned, population-based studies carried out using serovar information at specific institutions could provide valuable insights into the changing epidemiology of leptospirosis among dogs in the United States and Canada.

\section{Acknowledgements}

The assistance of Ms. Yun Shen, School of Veterinary Medicine, Purdue University, in performing VMDB searches is gratefully acknowledged.

\section{References}

Adin, C.A., Cowgill, L.D., 2000. Treatment and outcome of dogs with leptospirosis: 36 cases (1990-1998). J. Am. Vet. Med. Assoc. 216, 371-375.

American Public Health Association, 2000. Leptospirosis. In: Benenson, A.S. (Ed.), Control of Communicable Diseases Manual. American Public Health Association, Washington, DC, pp. 293-296.

Baldwin, C.J., Atkins, C.E., 1987. Leptospirosis in dogs. Compend. Contin. Educ. Pract. Vet. 9, 499-507.

Birnbaum, N., Center, S.A., Schermerhorn, T. et al., 1998. Naturally acquired leptospirosis in 36 dogs: serological and clinicopathological features. J. Small Anim. Pract. 39, 231-236.

Bolin, C.A., 1996. Diagnosis of leptospirosis: a reemerging disease of companion animals. Sem. Vet. Med. Surg. (Small Anim.) 11, 166-171.

Brown, C.A., Roberts, W., Miller, M.A. et al., 1996. Leptospira interrogans serovar grippotyphosa infection in dogs. J. Am. Vet. Med. Assoc. 209, 1265-1267.

Hanson, L.E., 1982. Leptospirosis in domestic animals: the public health. J. Am. Vet. Med. Assoc. 181, 15051509.

Harkin, K.R., Gartrell, C.L., 1996. Canine leptospirosis in New Jersey and Michigan 17 cases (1990-1995). J. Am. Anim. Hosp. Assoc. 32, 495-501.

Kahn, H.A., Sempos, C.T., 1989. Statistical Methods in Epidemiology. Oxford University Press, New York, pp. 95-105.

Kulldorff, M., 1997. A spatial scan statistic. Commun. Stat. Theory Meth. 26, 1481-1496.

Kulldorff, M., Athas, W.F., Feuer, E.J., Miller, B.A., Key, C.R., 1998a. Evaluating cluster alarms: a space-time scan statistic and brain cancer in Los Alamos. Am. J. Public Health 88, 1377-1380.

Kulldorff, M., Rand, K., Gherman, G., Williams, G., DeFrancesco, D., 1998b. SaTScan v2.1: Software for the Spatial and Space-Time Scan Statistics. National Cancer Institute, Bethesda, MD. http://dcp.nci.nih.gov/BB/ SaTScan.html.

Markey, B., 1995. Canine vaccination. Irish Vet. J. 48, 161-165.

Naus, J., 1965. The distribution of the size of maximum cluster of points on the line. J. Am. Stat. Assoc. 60, 532-538.

O’Brien, D.J., Kaneene, J.B., Getis, A., Lloyd, J.W., Swanson, G.M., Leader, R.W., 2000. Spatial and temporal comparison of selected cancers in dogs and humans, Michigan, USA, 1964-1994. Prev. Vet. Med. 47, 187-204. 
Paul, M.A., Wolf, A.M., 1999. Vaccination. What's right? What's not? In: Proceedings of the North American Veterinary Conference, 1999, pp. 20-22.

Prescott, J.F., Key, D., Osuch, M., 1999. Leptospirosis in dogs. Can. Vet. J. 40, 430-431.

Rentko, V.T., Clark, N., Ross, L.A., 1992. Canine leptospirosis: a retrospective study of 17 cases. J. Vet. Int. Med. 6, 235-244.

Ribotta, M., Fortin, M., Higgins, R., 2000. Canine leptospirosis: serology. Can. Vet. J. 41, 494-495.

Sykes, J.E., 2001. Canine leptospirosis-a re-emerging disease. In: Proceedings of the 19th Annual Meeting of the ACVIM, pp. 763-765.

Ward, M.P., Carpenter, T.E., 2000a. Techniques for analysis of disease clustering in space and in time in veterinary epidemiology. Prev. Vet. Med. 45, 257-284.

Ward, M.P., Carpenter, T.E., 2000b. Analysis of time-space clustering in veterinary epidemiology. Prev. Vet. Med. 43, 225-237.

Ward, M.P., Glickman, L.T.G., Guptill, L., 2002. Prevalence of and risk factors for leptospirosis among dogs in the United States and Canada. J. Am. Vet. Med. Assoc. 220, 53-58.

Wohl, J.S., 1996. Canine leptospirosis. Comp. Small Anim. 18, 1215-1224. 